\title{
The Protective Effect of Curcumin Against Cisplatin- Induced Nephrotoxicity Experimentally in Rats
}

\author{
Abd Alkareem Omar Maghmomeh ${ }^{2 *}$, Noha Abdel Rahman ${ }^{1}$ and Amal Mohamed El Gayar ${ }^{1}$ \\ ${ }^{1}$ Department of Biochemistry, University of Mansoura, Egypt \\ ${ }^{2}$ Department of Biochemistry, Delta University for Science and Technology, Egypt
}

*Corresponding author: Abd Alkareem Omar Maghmomeh, Department of Biochemistry, Faculty of Pharmacy, Delta University for science and technology, International Coastal Road, Gamasa city, Mansoura 35712, Egypt.

Received Date: November 20, 2019

Published Date: December 12, 2019

\begin{abstract}
The incidence of acute kidney injury (AKI) has been increasing over time. AKI increase the risk of progression of chronic kidney disease. Acute and chronic renal failures are global public health issues with different features to take into account in different parts of the world. Curcumin has renal protective properties against kidney damage. The exact mechanism of such protection is not clear. Therefore, this study was conducted to examine the molecular levels of the protective effect of curcumin on cisplatin induced nephrotoxicity in rats. We investigated the effects of curcumin in AKI which was induced in male Sprague Dawley rats using cisplatin $(4.5 \mathrm{mg} / \mathrm{kg}$ in 2 consecutive days). Curcumin was administered by oral tubes $200 \mathrm{mg} / \mathrm{kg}$ daily for 21 days. Serum creatinine, creatinine clearance, urea nitrogen, oxidative stress markers [glutathione (GSH), lipid peroxide (Malondialdehyde)] assessments of these parameters were carried out to indicate the effect of curcumin in AKI. Serum analysis revealed an alteration in parameters of kidney. A decrease in the antioxidant activity of kidney was recorded in cisplatin group while curcumin administration restored it. The results clarified the strong protective effect of curcumin on cisplatin induced nephrotoxicity in rats at the molecular levels.
\end{abstract}

Keywords: Acute kidney injury; Oxidative stress; Curcumin

\section{Introduction}

Acute kidney injury might occur with the use of several drugs, such as non-steroidal anti-inflammatory drugs, antibiotics, antineoplastic drugs and angiotensin-converting-enzyme inhibitors [1]. Severe, long, and repeated episodes of acute kidney injury increase the risk of progression of chronic kidney disease. Acute and chronic renal failure are global public health issues with different features to take into account in different parts of the world, renal complications, which involve most organ systems, can be treated and prevented, by using different therapeutic strategies [2]. Cisplatin is one of the most widely used and most effective cytotoxic agents in the treatment of a variety of malignant tumors, including lung, colorectal, ovarian, breast, head/neck, bladder, and testicular cancers in both children and adults [3]. On the other hand, cisplatin drugs have two main limitations: their severe side effects and the ability of cancers to develop drug resistance. Common side effects of cisplatin drugs include nausea, vomiting, and diarrhea, myelosuppression, neuropathy, ototoxicity, hepatotoxicity and nephrotoxicity [4]. Patients develop acute kidney injury [5] which may progress to chronic kidney injury [6]. Renal involvement is common after cisplatin injection (up to $30-50 \%$ of the cases), and it most often occurs in the second week of treatment [7]. Renal damage has a wide spectrum of sign symptoms e.g. hematuria, proteinuria, glucosuria, hypomagnesemia and most notably acute kidney injury [8]. Cisplatin-induced nephrotoxicity may range from mild and reversible structural alterations in tubular epithelial cells inducing a variable range of renal dysfunction (acute nephrotoxicity), to potentially irreversible renal failure leading to chronic and progressive renal insufficiency (chronic nephrotoxicity) [9]. Curcumin is a naturally occurring compound derived from the rhizomes of Curcuma longa. It is a member of the ginger family Zingiberaceae, found in the rhizome of the herb Curcuma longa, which is commonly known as turmeric [10]. Turmeric is widely used in therapeutic preparations [11]. Curcumin has been found to possess several properties including antioxidant [12], antimicrobial [13], antiviral [14], anti-inflammatory [15], anti-carcinogenic [16] and anti-diabetic [17]. Curcumin showed hepatoprotective activity 
against liver damage in animals induced by carbon tetrachloride [18]. In the present study, we tested curcumin in an in vivo model of cisplatin-induced kidney nephrotoxicity to assess its potential renoprotective effects.

\section{Materials and Methods}

\section{Chemicals and kits}

Curcumin was purchased from Sigma-aldrich (St. Louis, MO, USA). Creatinine, urea, malondialdehyde (MDA), reduced glutathione (GSH) and phosphate buffer saline (PBS) were purchased from Bio-diagnostic Co (Dokki, Giza, Egypt). Thiopental sodium was supplied in the form of (Anapental $500 \mathrm{mg} / \mathrm{vial}$ ), purchased from Sigma Tec Co., Egypt.

\section{Animals and experimental protocols}

This study comprised of 30 male Sprague Dawely rats 3 months old, whiting ( $225 \pm 25 \mathrm{gm}$ ), were utilized in the present study. Rats were housed in stainless steel rodent cages at room temperature $\left(25 \pm 2^{\circ} \mathrm{C}\right)$ and with 12 hours dark/light cycle and were provided with standard rat food and water. This study was carried out in strict accordance with the guidelines and authorization for the use of laboratory animals. The protocol was approved by the committee on the ethics of animal experiments of Faculty of Pharmacy, Mansoura University, for Animal Use. The rats were divided into 3 groups $(n=10)$ a) control group: rats were maintained on normal pellet diet. b) cisplatin group: rats were treated with was cisplatin (4.5 mg/ kg .day/i.p) for 2 consecutive days [19] c) cisplatin + curcumin: (4.5 mg/ kg .day) in 2 consecutive days [19], after the one day of cisplatin injection [20], rats were orally administered curcumin was suspended in (PBS) $(200 \mathrm{mg} / \mathrm{kg} /$ day $/$ ) for 21 days. The dose and duration were selected according to a previous study [21]. At the end of study, the rats were placed in a metabolic cage for 24 hours to collect 24-hour urine, and samples were taken for estimating urine creatinine [22]. After 12 hours of fasting rats were sacrificed after anesthetization using thiopental sodium (40 $\mathrm{mg} / \mathrm{kg}$, IP of $2.5 \%$ thiopental). Blood samples were withdrawn from thiopental-anesthetized animals via retro-orbital puncture after a fast of 12 hours [23]. Serum was extracted after blood centrifugation for $10 \mathrm{~min}$ at $4000 \times \mathrm{g}$. Kidney tissues were removed was homogenized in $5 \mathrm{ml}$ ice-cold PBS (0.02 M, pH 7.4) (10\% w/v), centrifuged at $3000 \mathrm{rpm}$ for $20 \mathrm{~min}$ at $4{ }^{\circ} \mathrm{C}$ and kept at $-80^{\circ} \mathrm{C}$ until further analysis.

\section{Determination of biochemical parameters}

Serum creatinine, urea, kidney malondialdehyde (MDA) and reduced glutathione (GSH) were assayed using calorimetric kits according to manufacturer's instructions.

\section{Statistical analysis}

Data were expressed as a mean \pm standard deviation $(M \pm S D)$ in each group. Statistical evaluations of the results were carried out by means of one-way analysis of variance (ANOVA), followed by Turkey multiple comparison tests. Statistical tests were performed using the Statistical Package for the Social Sciences (SPSS) version 13 (Chicago, IL, USA). Statistical significance was taken at $\mathrm{P}<0.05$ and $\mathrm{P}<0.01$. Graphing was carried out using GraphPad Prism software (Graphpad Software Inc., San Diego, USA).

\section{Results}

\section{Effect of curcumin on kidney function}

As shown in Figure 1-3, serum creatinine and urea levels were significantly increased, however, creatinine clearance was significantly reduced in Cis group when compared to the control group $(\mathrm{p}<0.01)$. Curcumin treatments were significantly reduced serum creatinine and urea levels and significantly increased creatinine clearance level compared to Cis group $(\mathrm{p}<0.05)$ (Figure $1-3)$.

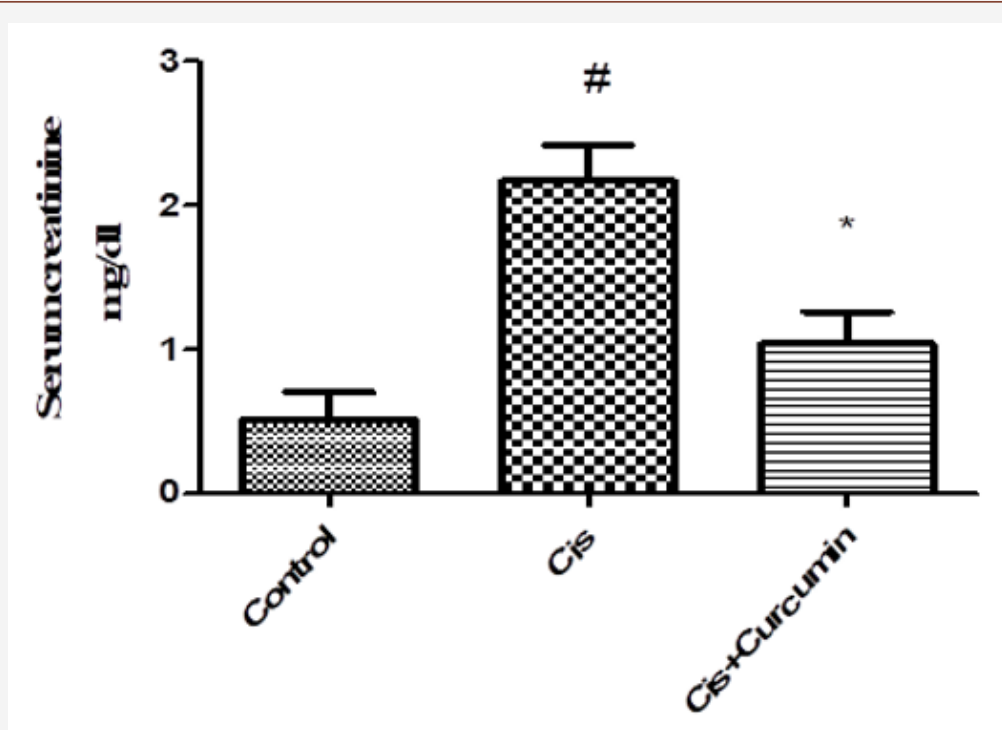

Figure 1: Effect of curcumin $(200 \mathrm{mg} / \mathrm{kg} /$ day, orally), on serum creatinine levels. Values are expressed as mean \pm SD. Symbols indicate statistical difference: \# : significant difference control group at $p<0.01 ;{ }^{*}$ : significant difference from nephrotoxicity group at $p<0.05$; Cis: Cisplatin. 


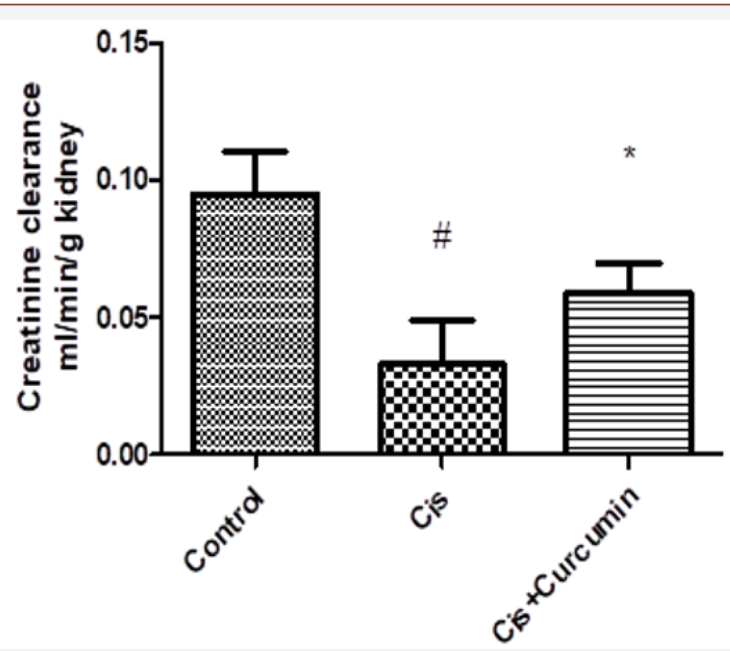

Figure 2: Effect of curcumin (200 mg/kg /day, orally), on creatinine clearance levels. Values are expressed as mean \pm SD. Symbols indicate statistical difference: \# : significant difference control group at $p<0.01$; ${ }^{*}$ : significant difference from nephrotoxicity group at $p<0.05$; Cis: Cisplatin.

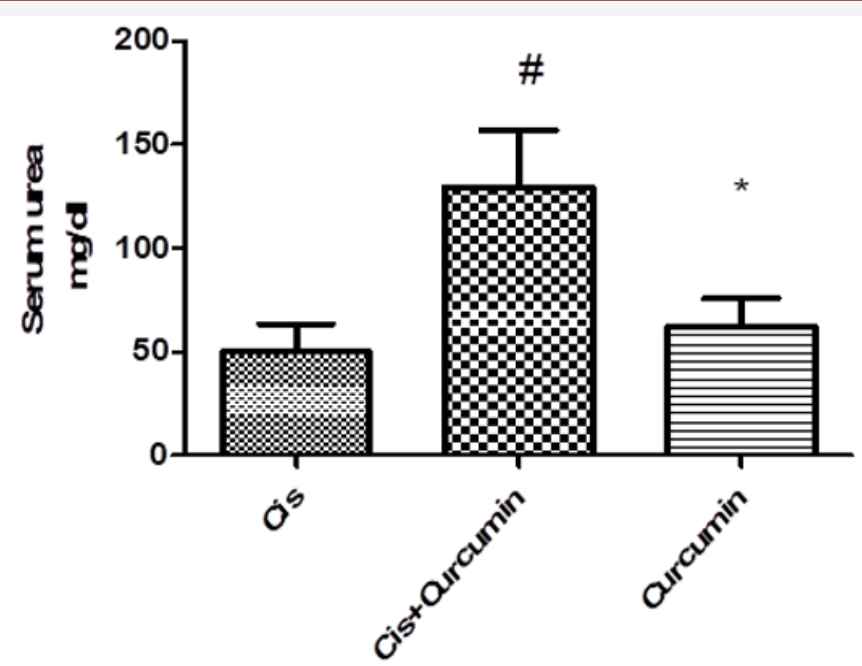

Figure 3: Effect of curcumin $(200 \mathrm{mg} / \mathrm{kg} /$ day, orally), on serum urea levels. Values are expressed as mean \pm SD. Symbols indicate statistical difference: \# : significant difference control group at $p<0.01$; ${ }^{*}$ : significant difference from nephrotoxicity group at $p<0.05$; Cis: Cisplatin.

\section{Effect of curcumin on oxidative stress}

As shown in Figure 4-5, kidney MDA was significantly increased however; GSH was significantly reduced in Cis group when compared to the control group ( $\mathrm{p}<0.01)$. Curcumin treatments were significantly reduced MDA and were significantly increased GSH compared to Cis group ( $\mathrm{p}<0.05)$ (Figure 4-5).

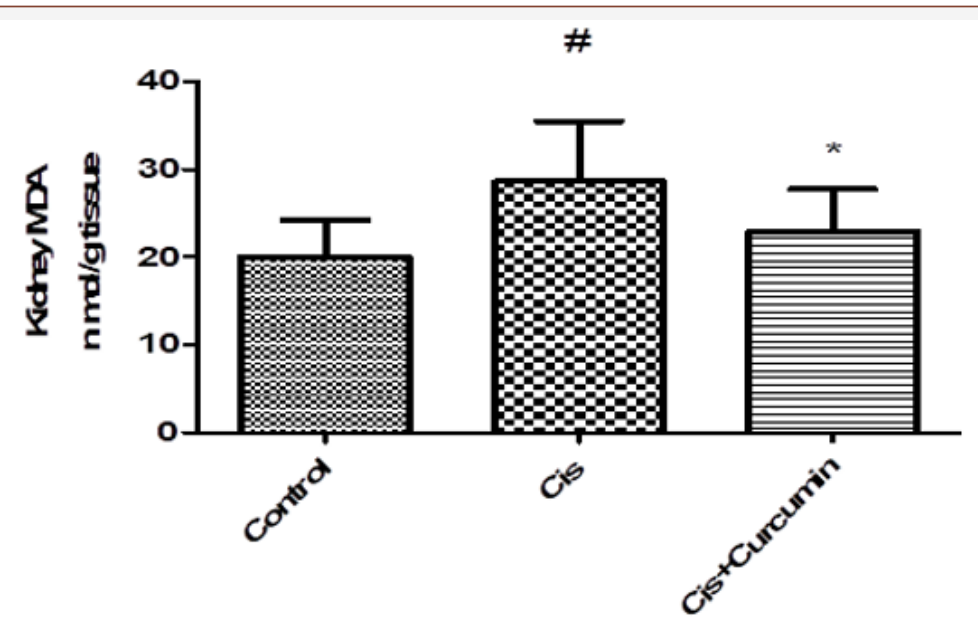

Figure 4: Effect of curcumin (200 mg/kg/day, orally), on Kidney MDA levels. Values are expressed as mean \pm SD. Symbols indicate statistical difference: \# : significant difference control group at $p<0.01 ;{ }^{*}$ : significant difference from nephrotoxicity group at $p<0.05$; Cis: Cisplatin; MDA: Malondialdehyde. 


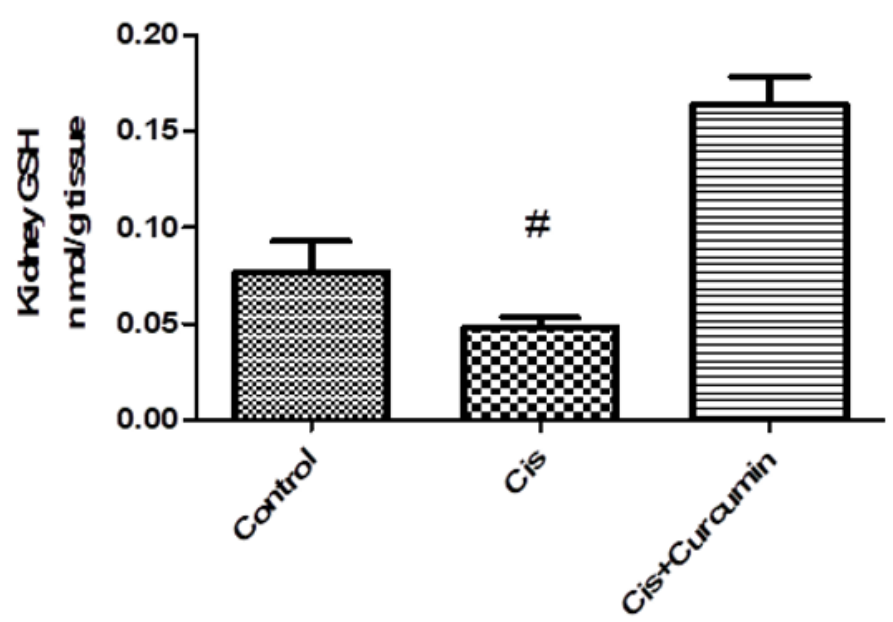

Figure 5: Effect of curcumin ( $200 \mathrm{mg} / \mathrm{kg} /$ day, orally), on Kidney GSH levels. Values are expressed as mean \pm SD. Symbols indicate statistical difference: \# : significant difference control group at $p<0.01{ }^{*}$ : significant difference from nephrotoxicity group at $p<0.05$; Cis: Cisplatin; GSH: Glutathione.

\section{Discussion}

Cisplatin is used to manage several human malignancies. However, nephrotoxicity is a serious side effect that limits its use as a chemotherapeutic agent. This may require reducing the dose or resorting to alternative treatment strategies [24]. Cisplatin induces glomerular and tubular dysfunctions [25] and injury of renal vasculature and structures [26]. Cisplatin causes tubular epithelial cell toxicity, vasoconstriction in the renal microvasculatureand inflammatory effects [27]. Cisplatin was also found to decrease glomerular filteration rate possibly due to its nephrotoxic effect on the S3 segment of the proximal tubule [28]. Patients develop acute kidney injury [5] which may progress to chronic kidney injury [6, 29]. In our study, serum and urine levels of creatinine and urea nitrogen were altered after cisplatin treatment indicating kidney injury. The evaluation of renal function and glomerular damage involved the examination of serum creatinine levels and reduction in creatinine clearance in accordance with previous reports [30]. Creatinine is a waste product of metabolism excreted by the kidneys without reabsorption. Hence, it is used as an index of renal function and to monitor renal dysfunction or damage [31]. Our study showed that treatment with cisplatin for $(4.5 \mathrm{mg} / \mathrm{kg}$. day) rats' group [19] two days was sufficient to induce nephrotoxicity in rats [20] as indicated by elevated serum creatinine and reduced creatinine clearance. Curcumin was found to mitigate cisplatin-induced kidney injury in pretreated rats as evidenced by reduction in serum creatinine and blood urea nitrogen. This is in agreement with previous studies [32]. Curcumin treatment ameliorated the former parameters. Creatinine and urea levels decreased significantly whereas creatinine clearance levels increased significantly during the treatment when compared with the nephrotoxicity groups. Oxidative stress plays a key role in cisplatin-induced renal dysfunction. Cisplatin enhances the production of superoxide, peroxynitrite, hydrogen peroxide and hydroxyl radicals via mobilization of iron from renal cortical mitochondria [22]. The enhanced expression of reactive oxygen species (ROS) by cisplatin is directly related to tubulointerstetial fibrosis [33]. Cisplatin is activated when it enters the cell through binding to the N7 reactive center on purine rings, resulting in DNA damage in cancer cells, blocking cell division and contributing to apoptotic cell death [34]. Cisplatin also induces ROS-mediated cell death [35]. ROS production increases during cisplatin treatment of cultured renal tubular cells, kidney slices, and in vivo in whole animals [36].

\section{Conclusion}

As a result of increased ROS production by cisplatin, antioxidant enzymes such as GSH are depleted [37]. Cisplatin also triggers MDA production in renal tissue [30]. MDA is a reactive aldehyde and forms covalent protein adducts which are referred to as advanced lipoxidation end products, very similar to advanced glycation end products $[38,39]$ It is the product of lipid peroxidation and reflects the degree of oxidation in the body. GSH is a tripeptide abundant in cells and is responsible for modulation of cell proliferation, antioxidant defense and detoxification of drugs and toxins. GSH also prevents damage to important cellular components caused by reactive oxygen species such as free radicals, peroxides, lipid peroxides and heavy metals [40]. Cisplatin can produce ROS through different mechanisms. Cisplatin can rapidly react with thiolcontaining molecules, primarily glutathione [41]. Once glutathione and other antioxidants are consumed, a shift in the cellular redox status occurs. ROS then accumulates within the cell and causing a state of oxidative stress. [42]. ROS may also disrupt the respiratory chain and cause mitochondrial dysfunction. The cytochrome P450 system has also been reported to be responsible for generating ROS as demonstrated in in vitro and in vivo models [43, 44]. In this study, the antioxidant status was assessed by measuring GSH and MDA levels. Kidney MDA was significantly increased in cisplatin group compared to control group. However, GSH was significantly reduced in the cisplatin group when compared to the control group. Curcumin treatment significantly reduced MDA and were significantly increased GSH compared to cisplatin group $(\mathrm{p}<0.05)$. 
Curcumin is known for its high oxygen-radical scavenging and quenching power [12]. It is a scavenger of free oxygen radicals and stimulates the activity of additional antioxidant molecules such as superoxide dismutase, catalase, and glutathione peroxidase [45]. It is a bifunctional antioxidant [46] because of its ability to react directly with reactive species and to induce an up regulation of various cytoprotective and antioxidant proteins [47]. Curcumin can react with ROS through its phenolic and methoxy groups and it is thought to be one of the mechanisms through which it can protect the renal epithelial from ROS activity [48]. Curcumin can indirectly induce the expression of cytoprotective proteins such as superoxide dismutase (SOD), catalase (CAT) [49], GSH [50], nicotinamide adenine dinucleotide phosphate (NADPH), quinone oxidoreductase 1 (NQ01) [51]. Furthermore, it has been reported that curcumin can increase the synthesis and concentration of GSH [52]. In summary, this study demonstrated that curcumin can protect against nephrotoxic effects of cisplatin in rats. The protective effect of curcumin occurred through the up regulation of antioxidants and suppression of oxidative stress markers. Curcumin is a promising therapy for management of kidney nephrotoxicity. Further in vitro studies are needed to outline the signaling pathways involved in curcumin actions during nephrotoxicity.

\section{Acknowledgement}

None.

\section{Conflict of Interest}

All authors declare no potential conflict of interest including any financial, personal or other relationships with other people or organizations within this work

\section{References}

1. Coca SG, Singanamala S, CR Parikh (2012) Chronic kidney disease after acute kidney injury: a systematic review and meta-analysis. Kidney Int 81(5): 442-448.

2. Trujillo J, Chirino Y, Molina Jijón E, Andérica Romero AC, Tapia E, et al. (2013) Renoprotective effect of the antioxidant curcumin: Recent findings. Redox Biol 17(1): 448-456.

3. Cerri S, Piccolini VM, Santin G, Bottone MG, De Pascali SA, et al. (2011) The developmental neurotoxicity study of platinum compounds. Effects of cisplatin versus a novel Pt (II) complex on rat cerebellum. Neurotoxicol Teratol 33(2): 273-281.

4. Di Francesco AM, A Ruggiero, R Riccardi (2002) Cellular and molecular aspects of drugs of the future: oxaliplatin. Cell Mol Life Sci 59(11): 19141927.

5. Chawla LS, Eggers PW, Star RA, Kimmel PL (2014) Acute kidney injury and chronic kidney disease as interconnected syndromes. N Engl J Med 371(1): 58-66

6. Uccelli A, L Moretta, V Pistoia (2008) Mesenchymal stem cells in health and disease. Nature Reviews Immunology 8(9): 726-736.

7. Engle JE (1990) Clinical physiology of acid-base and electrolyte disorders. JAMA 263(17): 2375-2376.

8. Ciarimboli G, Ludwig T, Lang D, Pavenstädt H, Koepsell H, et al. (2005) Cisplatin nephrotoxicity is critically mediated via the human organic cation transporter 2. Am J Pathol 167(6): 1477-1484.

9. Kang DG, Lee AS, Mun YJ, Woo WH, Kim YC et al. (2004) Butein ameliorates renal concentrating ability in cisplatin-induced acute renal failure in rats. Biol Pharm Bull 27(3): 366-370.
10. Altenburg JD, Bieberich AA, Terry C, Harvey KA, Vanhorn JF, et al. (2011) A synergistic antiproliferation effect of curcumin and docosahexaenoic acid in SK-BR-3 breast cancer cells: unique signaling not explained by the effects of either compound alone. BMC cancer 11(1): 149.

11. Manolova Y, Deneva V, Antonov L, Drakalska E, Momekova D, et al. (2014) The effect of the water on the curcumin tautomerism: A quantitative approach. Spectrochimica Acta Part A: Molecular and Biomolecular Spectroscopy 132: 815-820.

12. Al Jassabi S, KA Ahmed, MA Abdulla (2012) Antioxidant effect of curcumin against microcystin-LR-induced renal oxidative damage in Balb/c mice. Tropical Journal of Pharmaceutical Research 11(4): 531536.

13. S Tajbakhsh, K Mohammadi, I Deilami, K Zandi, M Fouladvand, et al. (2008) Antibacterial activity of indium curcumin and indium diacetylcurcumin. African Journal of Biotechnology 7(21).

14. Bereswill S, Muñoz M, Fischer A, Plickert R, Haag LM, et al. (2010) Antiinflammatory effects of resveratrol, curcumin and simvastatin in acute small intestinal inflammation. PloS one 5(12): e15099.

15. Kutluay SB, Doroghazi J, Roemer ME, Triezenberg SJ (2008) Curcumin inhibits herpes simplex virus immediate-early gene expression by a mechanism independent of p300/CBP histone acetyltransferase activity. Virology 373(2): 239-247.

16. Das L, M Vinayak (2012) Anti-carcinogenic action of curcumin by activation of antioxidant defence system and inhibition of NF- $\mathrm{kB}$ signalling in lymphoma-bearing mice. Bioscience reports 32(2): 161170.

17. Aziz MTA, Ibrahim NEI, Dimitri PM, Ameen MR, Mohamed AAW, et al. (2013) Signaling mechanisms of a water-soluble curcumin derivative in experimental type 1 diabetes with cardiomyopathy. Diabetology \& metabolic syndrome 5(1): 13.

18. Somanawat K, D Thong Ngam, N Klaikeaw (2013) Curcumin attenuated paracetamol overdose induced hepatitis. World Journal of Gastroenterology 19(12): 1962-1967.

19. Wu HH, Jia HR, Zhang Y, Liu L, Xu DB, et al. (2015) Monitoring the progression of renal fibrosis by $\mathrm{T} 2$-weighted signal intensity and diffusion weighted magnetic resonance imaging in cisplatin induced rat models. Chin Med J (Engl) 128(5): 626-631.

20. Elhusseini FM, Saad MA, Anber N, Elghannam D, Sobh MA, et al. (2016) Long Term Study of Protective Mechanisms of Human Adipose Derived Mesenchymal Stem Cells on Cisplatin Induced Kidney injury in SpragueDawley Rats. J Stem Cells Regen Med 12(1): 36-48.

21. Lian N, Jiang Y, Zhang F, Jin H, Lu C, et al. (2015) Curcumin regulates cell fate and metabolism by inhibiting hedgehog signaling in hepatic stellate cells. Lab Invest 95(7): 790-803.

22. Kuhad A, Tirkey N, Pilkhwal S, Chopra K (2006) Renoprotective effect of Spirulina fusiformis on cisplatin-induced oxidative stress and renal dysfunction in rats. Renal failure 28(3): 247-254.

23. Sharma A, Fish BL, Moulder JE, Medhora M, Baker JE, et al. (2014) Safety and blood sample volume and quality of a refined retro-orbital bleeding technique in rats using a lateral approach. Lab Anim (NY) 43(2): 63-66.

24. Taguchi T, Nazneen A, Abid MR, Razzaque MS (2005) Cisplatin-associated nephrotoxicity and pathological events. Contrib Nephrol 148: 107-121.

25. Ikarashi Y, Kakihara T, Imai C, Tanaka A, Watanabe A, et al. (2004) Glomerular dysfunction, independent of tubular dysfunction, induced by antineoplastic chemotherapy in children. Pediatr Int 46(5): 570-575.

26. Kintzel PE (2001) Anticancer drug-induced kidney disorders. Drug Saf 24(1): $19-38$

27. Pezeshki Z, Nematbakhsh M, Mazaheri S, Eshraghi Jazi F, Talebi A, et al. (2012) Estrogen Abolishes Protective Effect of Erythropoietin against Cisplatin-Induced Nephrotoxicity in Ovariectomized Rats. ISRN Oncol 890310.

28. Dobyan DC, Levi J, Jacobs C, Kosek J, Weiner MW (1980) Mechanism of cis-platinum nephrotoxicity: II. Morphologic observations. J Pharmacol Exp Ther 213(3): 551-556. 
29. Perse M, Z Veceric Haler (2018) Cisplatin-Induced Rodent Model of Kidney Injury: Characteristics and Challenges. Biomed Res Int 1462802.

30. Niles DJ, Gordon JW, Huang G, Reese S, Adamson EB, et al. (2018) Evaluation of renal metabolic response to partial ureteral obstruction with hyperpolarized ${ }^{13} \mathrm{C}$ MRI. NMR Biomed 31(1).

31. Guyton A, J Hall (2006) The body fluids and kidneys. Textbook of medical physiology 6: 339.

32. He L, Peng X, Zhu J, Liu G, Chen X, et al. (2015) Protective effects of curcumin on acute gentamicin-induced nephrotoxicity in rats. Can J Physiol Pharmacol 93(4): 275-282.

33. Kawai Y, Satoh T, Hibi D, Ohno Y, Kohda Y et al. (2009) The effect of antioxidant on development of fibrosis by cisplatin in rats. J Pharmacol Sci 111(4): 433-439.

34. Dasari S, PB Tchounwou (2014) Cisplatin in cancer therapy: molecular mechanisms of action. European journal of pharmacology 740: 364-378.

35. Brozovic A, Ambriovic RA, M Osmak (2010) The relationship between cisplatin-induced reactive oxygen species, glutathione, and BCL-2 and resistance to cisplatin. Crit Rev Toxicol 40(4): 347-359.

36. Kawai Y, Satoh T, Hibi D, Ohno Y, Kohda Y, et al. (2009) The effect of antioxidant on development of fibrosis by cisplatin in rats. Journal of pharmacological sciences 111(4): 433-439.

37. Sharma S, A Joshi, S Hemalatha (2017) Protective Effect of Withania coagulans Fruit Extract on Cisplatin-induced Nephrotoxicity in Rats. Pharmacognosy Research 9(4): 354-361.

38. Farmer EE, Davoine C (2007) Reactive electrophile species. Curr Opin Plant Biol 10(4): p. 380-386.

39. Laila AE, Nehal ME, Abdalkareem OM (2017) Effect of 2-hydroxychalcone on adiponectin level in type 2 diabetes induced experimentally in rats 4(1): $1-8$.

40. Forman HJ, H Zhang, A Rinna (2009) Glutathione: overview of its protective roles, measurement, and biosynthesis. Mol Aspects Med 30(1-2): 1-12.

41. Siddik ZH (2003) Cisplatin: mode of cytotoxic action and molecular basis of resistance. Oncogene 22(47): 7265-7279.
42. Liu H, R Baliga (2003) Cytochrome P450 2E1 null mice provide novel protection against cisplatin-induced nephrotoxicity and apoptosis. Kidney international 63(5): 1687-1696.

43. Baliga R, Zhang Z, Baliga M, Ueda N, Shah SV (1998) In vitro and in vivo evidence suggesting a role for iron in cisplatin-induced nephrotoxicity. Kidney international 53(2): 394-401.

44. Pabla N, Z Dong (2008) Cisplatin nephrotoxicity: mechanisms and renoprotective strategies. Kidney international 73(9): 994-1007.

45. Topcu Tarladacalisir Y, M Sapmaz Metin, T Karaca (2016) Curcumin counteracts cisplatin-induced nephrotoxicity by preventing renal tubular cell apoptosis. Ren Fail 38(10): 1741-1748.

46. Dinkova Kostova AT, P Talalay (2008) Direct and indirect antioxidant properties of inducers of cytoprotective proteins. Mol Nutr Food Res 52(1): p. S128-S138.

47. Ak T, I Gulcin (2008) Antioxidant and radical scavenging properties of curcumin. Chem Biol Interact 174(1): 27-37.

48. Cohly HH, Taylor A, Angel MF, Salahudeen AK (1998) Effect of turmeric, turmerin and curcumin on H2O2-induced renal epithelial (LLC-PK1) cell injury. Free Radic Biol Med 24(1): 49-54

49. Panchal HD, Vranizan K, Lee CY, Ho J, Ngai J, et al. (2008) Early antioxidative and anti-proliferative curcumin effects on neuroglioma cells suggest therapeutic targets. Neurochem Res 33(9): 1701-1710.

50. Yarru LP, Settivari RS, Gowda NK, Antoniou E, Ledoux DR, et al. (2009) Effects of turmeric (Curcuma longa) on the expression of hepatic genes associated with biotransformation, antioxidant, and immune systems in broiler chicks fed aflatoxin. Poult Sci 88(12): 2620-2627.

51. Ye SF, Hou ZQ, Zhong LM, Zhang QQ (2007) Effect of curcumin on the induction of glutathione S-transferases and NADP $(\mathrm{H})$ : quinone oxidoreductase and its possible mechanism of action. Yao Xue Xue Bao 42(4): 376-380.

52. Lavoie S, Chen Y, Dalton TP, Gysin R, Cuénod M, et al. (2009) Curcumin, quercetin, and tBHQ modulate glutathione levels in astrocytes and neurons: importance of the glutamate cysteine ligase modifier subunit. J Neurochem 108(6): 1410-1422. 